\title{
Identification of Long Non-Coding RNAs Involved in Fat Deposition in Pigs Using Two High-Throughput Sequencing Methods
}

\section{Yibing Liu}

China Agricultural University

Hong Ao

Chinese Academy of Agricultural Sciences

Fengxia Zhang

China Agricultural University

\section{Xitong Zhao}

China Agricultural University

\section{Huatao Liu}

China Agricultural University

\section{Yong Shi}

China Agricultural University

Kai Xing ( $\nabla$ xk181986@163.com )

Beijing University of Agriculture

\section{Ying Yu}

China Agricultural University

Chuduan Wang

China Agricultural University

\section{Research}

Keywords: IncRNA, fat deposition, pig, RNA-seq

Posted Date: September 8th, 2020

DOI: https://doi.org/10.21203/rs.3.rs-70892/v1

License: (c) (i) This work is licensed under a Creative Commons Attribution 4.0 International License.

Read Full License 


\section{Identification of long non-coding RNAs involved in fat deposition in pigs using two high-throughput sequencing methods}

Yibing Liu ${ }^{1}$, Hong Ao ${ }^{3}$, Fengxia Zhang ${ }^{1}$, Xitong Zhao ${ }^{1}$, Huatao Liu $^{1}$, Yong Shi ${ }^{1}$, Kai Xing ${ }^{2 *}$, Ying

$\mathrm{Yu}^{1 *}$, Chuduan Wang ${ }^{1 *}$

1. Key Laboratory of Animal Genetics, Breeding and Reproduction, Ministry of Agriculture,

National Engineering Laboratory for Animal Breeding, College of Animal Science and

Technology, China Agricultural University, Beijing, China

2. Animal Science and Technology College, Beijing University of Agriculture, Beijing, China

3. Institute of Animal Science, Chinese Academy of Agricultural Sciences, Beijing, China)

Email addresses:

Yibing Liu, 279547013@qq.com

Kai Xing, xk181986@163.com

Hong Ao, aohong@ caas.cn

Fengxia Zhang, 895315294@qq.com

Xitong Zhao, zhaoxitong0348@163.com

Huatao Liu, 925457179@qq.com

Yong Shi, 2273190789@qq.com

Ying Yu, yuying@cau.edu.cn

Chuduan Wang, cdwang@cau.edu.cn 
Abstract:

Background: Adipose is an important body tissue in pigs, and fatty traits are critical in pig production. The function of long non-coding RNA (lncRNA) in fat deposition and metabolism has been proven in previous studies. In this study, we focused on lncRNAs associated with fattening traits in pigs. The adipose tissue of six Landrace pigs with either extremely-high or -low backfat thickness $\left(n_{\text {high }}=3, n_{\text {low }}=3\right.$ ) was collected, after which we performed strand-specific RNA sequencing using biological replicates and pooling methods.

Results: A total of 19,631 genes and 2,013 lncRNAs were identified using the coding potential calculator, coding-non-coding index, and Pfam database, including 334 known transcripts and 1,679 novel transcripts. Using edgeR, we determined that $220 \operatorname{lncRNAs}$ and 1,512 genes were differentially expressed $(\mid$ Fold Change $\mid>2$ and false discovery rate $<0.05)$ between the two groups in biological replicate RNA sequencing (RNA-seq), and 127 lncRNAs and 2,240 genes were differently expressed in pooling RNA-seq. Further Kyoto Encyclopedia of Genes and Genomes and Gene Ontology enrichment analysis of the differentially expressed genes found that some of the genes were involved in several key pathways related to fat development. After targeting gene prediction, we determined that some cis-target genes of the differentially expressed lncRNAs play an important role in fat deposition. For example, ACSL3 is cis-targeted by lncRNA TCONS-00052400, and it can activate the conversion of long-chain fatty acids. In addition, IncRNA TCONS_00041740 was up-regulated in the high backfat thickness group, and its cis-target gene $A C A C B$ was also up-regulated in this group. It has been reported that $A C A C B$ is the rate-limiting enzyme in fatty acid oxidation.

Conclusions: Since these genes have necessary functions in fat metabolism, the results imply that 
the lncRNAs detected in our study may affect fat deposition in pigs through regulation of their target genes. In summary, our study explored the regulation of lncRNA and their target genes on fat deposition in pigs and provided new insights for further investigation of the biological functions of lncRNA.

Keywords: IncRNA, fat deposition, pig, RNA-seq

\section{Introduction}

Fat deposition is an important biological process in pig growth. Fat traits are closely related to pork quality as well as the production efficiency and reproductive traits of pigs ${ }^{[1]}$. The fat content of pigs also affects consumers' choice of pork ${ }^{[2]}$. Furthermore, pigs are an ideal animal model for human disease research ${ }^{[3]}$ on various diseases such as type 2 diabetes ${ }^{[4]}$, hypertension, and coronary heart disease ${ }^{[5]}$. Determining the molecular markers that affect subcutaneous fat deposition in pigs is an important way to effectively accelerate its genetic improvement. Therefore, it is of great significance to explore the molecular regulation mechanisms of fat deposition in pigs.

Fat deposition is a complex process influenced by multiple genes and epigenetic regulators including long non-coding RNA (lncRNA). LncRNA is a type of non-coding RNA with a transcript length greater than $200 \mathrm{nt}$, and accounts for approximately $80 \%$ of mammalian genomic transcription products ${ }^{[6]}$. LncRNA can regulate the expression of target genes through a variety of methods and plays an important role in complex biological processes in the body. Many studies have shown that lncRNA can activate gene transcription at the transcriptional level ${ }^{[7]}$, participate in translation substitution at the post-transcriptional level ${ }^{[8]}$, and produce imprinted genes at the epigenetic modification level ${ }^{[9]}$. 
With the rapid development of high-throughput sequencing technology, transcriptome sequencing has been widely used in animal studies. In recent years, there have been an increasing number of studies on lncRNA function using high-throughput sequencing technology. The regulation of IncRNA on fat deposition has been reported in various species, including humans, mice, and cattle. For example, Alvarez used de novo RNA sequencing to compare the transcriptomes of different adipose tissues in mice and identified 127 lncRNAs from brown adipose tissue. In addition, they identified lnc-BATE1, which can regulate the growth and thermogenesis of brown adipocytes ${ }^{[10]}$. Li sequenced preadipocytes and mature adipocytes of Qinchuan cattle and discovered 16 differentially expressed lncRNAs. Among them, lncRNA-ADNCR can inhibit the differentiation of adipocytes by competitively binding to miR-204 with SIRT1 ${ }^{[11]}$. However, there are few studies that focus on the regulation of lncRNA on backfat deposition in pigs, and the molecular regulation mechanism for this process is still unclear.

In this study, we performed biological replicates and two pools using high/low phenotype strand-specific library RNA sequencing (RNA-seq) on six Landrace pigs with either extremely-high or -low backfat thickness. We identified the differentially expressed genes and IncRNAs, combining two-part results to obtain the key genes and lncRNAs that affect fat deposition in Landrace pigs (Figure 1). In addition, we explored the crucial genes and lncRNAs involved in molecular regulation mechanisms to provide information for improving fat deposition in pigs. 
A Landrace female pig resource population was used in this study. We used real-time B-mode ultrasonography (HS1500 convex scanner; Honda Electronics, Toyohashi, Japan) to measure the backfat thickness between the last 3rd and 4th ribs. Combined with pedigree information, we selected three full-sib Landrace pairs with extreme backfat thickness from 132 female Landrace pigs. Three of the pigs with extremely-high backfat thickness formed the high group (BH, $8.88 \pm$ $0.80 \mathrm{~mm}$ ) and three with extremely-low backfat thickness formed the low group (BL, $3.58 \pm 0.39$ $\mathrm{mm})$. The experimental population phenotype and selection criteria were the same as those previously reported ${ }^{[12,13]}$. The subcutaneous fat tissues of these individuals were collected after slaughter and then immediately stored in liquid nitrogen until use.

\section{RNA extraction and sequencing}

We used a total RNA extraction kit (Bioteke Corporation, Wuxi, China) to extract the total RNAs of all the samples according to the manufacturer's recommendations, and the quality of the extract was checked by a 2100 Bioanalyzer (Agilent Technologies, Santa Clara, CA, USA). After quality control, a library was prepared using the total RNAs. The sequencing library was prepared by random fragmentation of the cDNA samples, followed by 5' and 3' adapter ligation. The adapter-ligated fragments were then PCR-amplified and gel-purified. For cluster generation, the library was loaded into a flow cell where fragments were captured on a lawn of surface-bound oligos complementary to the library adapters. Each fragment was amplified into distinct clonal clusters through bridge amplification. After cluster generation, the samples were sequenced in an Illumina Hiseq 2500 sequencer (Illumina, Inc., San Diego, CA, USA). The sequencing was performed by Biomarker Technologies, Inc. (Rohnert Park, CA, USA). The raw data obtained from the sequencing were trimmed using Trimmomatic software ${ }^{[14]}$. Clean data were achieved 
111

112

after deleting the adapter and low quality reads (Phred quality score $\leqslant 10$ ). Using FastQC (v 0.11.8) ${ }^{[15]}$ to check the quality of the paired-end reads of all the six samples. We then used a NGSQC Toolkit (v 2.3.3) ${ }^{[16]}$ to perform quality inspection and data filtering of the high-throughput sequencing data. Only good quality trimmed reads were considered for downstream analysis.

\section{Mapping and assembly of sequenced RNA reads}

The pig reference genome (Sus scrofa 11.1) and annotation files were downloaded from the Ensembl database (http://asia.ensembl.org/Sus_scrofa/Info/Index) and indexed with Bowtie2 (v 2.3.0) ${ }^{[17]}$. Clean reads were aligned with the reference genome using TopHat2 (v 2.1.1) ${ }^{[18]}$ alignment program. Software Cufflinks (v 2.2.1) ${ }^{[19]}$ was used to assemble the mapped reads and determine their abundance in all the samples, keeping all parameters as default. Cuffmerge, a Cufflinks suite tool, was used to merge samples and obtain the merged gene transfer format (gtf) file for further downstream analysis.

\section{LncRNA filtering pipeline}

High stringency was useed to filter out putative lncRNAs from the RNA-Seq assembled transcripts. Only non-coding classcodes ( the Cuffmerge results; all others were removed. Transcripts with less than 200 nucleotides and less than two exon numbers were also deleted. Filtered transcripts were subjected to three of the most frequently used tools for identifying long non-coding RNAs, two of which were the Coding Potential Calculator (CPC) ${ }^{[20]}$ and Coding-Non-Coding-Index (CNCI) ${ }^{[21]}$. Common transcripts from the results of these two tools were then scanned against the Pfam database to discover any probable protein domains ${ }^{[22]}$. Similar protocols have been used in previous studies to identify 
$\operatorname{lncRNAs}{ }^{[9,23]}$.

\section{Identification of differentially and exclusively expressed IncRNA and mRNA}

We used htseq software (v 0.7.2) ${ }^{[24]}$ to count the read counts of genes and lncRNAs, and further screened genes by their expression level in different groups. EdgeR ${ }^{[25]}$, an $\mathrm{R}$ package, was used to determine the expression difference between the two groups using an overdispersed Poisson model. We used false discovery rate $($ FDR $) \leqslant 0.05$, and $\mid$ Fold Change $\mid>2$ as the threshold to identify significantly differentially expressed lncRNAs (DELs) and differentially expressed genes (DEGs). Heatmap clustering analysis of all differentially expressed lncRNAs and messenger RNAs (mRNAs) was calculated using the pheatmap $\mathrm{R}^{[26]}$ package. Moreover, if the transcripts were expressed in more than two samples in one group and were not expressed in all samples of the other group, we considered them to be exclusively expressed lncRNAs and exclusively expressed genes.

\section{Gene ontology and KEGG pathway functional annotations}

Gene Ontology (GO, http://www.geneontology./org ${ }^{[27]}$ ) is the international standard classification of gene function. It classifies the function of genes along three aspects, including molecular function, biological process, and cellular component. Gene Ontology (GO) provides a framework and set of concepts for describing the functions of gene products from all organisms. The Kyoto Encyclopedia of Genes and Genomes (KEGG, http://www.genome.jp/kegg ${ }^{[28]}$ ) can systematically analyze gene function and is a genomic information database that helps researchers to study genes and expression information as a whole network. The functional annotation of the DEGs in this study based on GO and KEGG was completed using DAVID (v 6.8) ${ }^{[29]}$ and KOBAS 3.0 [30], respectively. The GO terms and KEGG pathways with $\mathrm{p}$ values $<0.05$ were considered as 
155

156

157

significantly enriched, and the results were plotted using the gglot $2{ }^{[31]} \mathrm{R}$ package.

\section{Target genes prediction and functional annotations}

To investigate the function of the differentially expressed lncRNAs, we searched their nearby genes and considered them as potential targets of IncRNAs. It has been reported that the main function of lncRNA is to regulate protein-coding genes through cis- and trans-regulation ${ }^{[32,33]}$. Bedtools (v2.25.0) ${ }^{[34]}$ was used to search neighborhood genes around $100 \mathrm{~kb}$ upstream and downstream of the DELs. In addition, we examined the Spearman's rank correlation between the expression levels of the DELs and DEGs to predict their targeting relationship.

\section{Statistical Analysis}

All data were reported as mean \pm standard error of mean. The functions glmFit() and glmLRT() of the edgeR package were used to perform a negative binomial generalized log-linear model fitting and significance test, respectively, and we used the FDR method to adjust the p values. An FDR < 0.05 was considered significant.

\section{Results}

\section{Overview of the IncRNA sequencing data}

In the biological replicate RNA-seq, a total of $16.24,16.50,16.20,16.32,16.07$, and $17.34 \mathrm{~Gb}$ of clean data were generated in the six samples, respectively. Furthermore, the results of the pooling RNA-seq were $16.45 \mathrm{~Gb}$ and $15.16 \mathrm{~Gb}$ in the extremely-high (HIGH) and extremely-low (LOW) backfat thickness groups, respectively. The average of the guanine-cytosine (GC) content was between $49.37 \%$ and $55.55 \%$. The Q30 ranged from $92.53 \%$ to $94.27 \%$. These results show that 
177 the quality of our eight libraries was suitable for subsequent data analysis.

178 Next, the clean reads were aligned to the reference genome (Sus scrofa 11.1) using Tophat v2.1.1.

179 In each sample, more than $77.82 \%$ of the clean reads were uniq mapped. The summary of the

180 sequencing data is shown in Table 1. 
Table 1 Summary of reads after quality control and mapping to the reference genome

\begin{tabular}{|c|c|c|c|c|c|c|c|c|}
\hline \multirow[b]{2}{*}{ Sample ID } & \multicolumn{6}{|c|}{ Biological Replicate RNA sequencing } & \multicolumn{2}{|c|}{ Pooling RNA sequencing } \\
\hline & H22511 & H23712 & H31210 & L22509 & L23709 & L31208 & HIGH & LOW \\
\hline Clean Data & 16202102282 & 16317045132 & 17343934922 & 16244957328 & 16074210300 & 16498461484 & 15526646169 & 14298214979 \\
\hline $\mathrm{GC}(\%)$ & 50.71 & 54.34 & 55.24 & 50.89 & 55.55 & 51.63 & 51.38 & 49.37 \\
\hline Q30(\%) & 94.27 & 93.32 & 93.71 & 93.76 & 93.84 & 93.76 & 92.53 & 92.62 \\
\hline Total Reads & 108162730 & 109081084 & 115964182 & 109091500 & 107499706 & 110216006 & 106068646 & 97723146 \\
\hline \multirow{2}{*}{ Mapped Reads } & 103261178 & 99528016 & 106878278 & 101789248 & 96471336 & 103862050 & 84998524 & 79213694 \\
\hline & $(95.47 \%)$ & $(91.24 \%)$ & $(92.16 \%)$ & $(93.31 \%)$ & $(89.74 \%)$ & $(94.23 \%)$ & $(80.14 \%)$ & $(81.06 \%)$ \\
\hline \multirow{2}{*}{ Uniq Mapped Reads } & 96976910 & 93090379 & 97146560 & 97935676 & 92128163 & 98096189 & 82541284 & 77406164 \\
\hline & $(89.66 \%)$ & $(85.34 \%)$ & $(83.77 \%)$ & $(89.77 \%)$ & $(85.70 \%)$ & $(89.00 \%)$ & $(77.82 \%)$ & $(79.21 \%)$ \\
\hline \multirow{2}{*}{ Multiple Mapped Reads } & 6284268 & 6437637 & 9731718 & 3853572 & 4343173 & 5765861 & 2457240 & 1807530 \\
\hline & $(5.81 \%)$ & $(5.90 \%)$ & $(8.39 \%)$ & $(3.53 \%)$ & $(4.04 \%)$ & $(5.23 \%)$ & $(2.32 \%)$ & $(1.85 \%)$ \\
\hline
\end{tabular}

Guanine-cytosine (GC) (\%) is the percentage of G and C bases in the total nucleotides; Q30 (\%) is the percentage of the bases' mass greater than or equal to Q30 in the clean data; Total Reads is the number of clean reads; Uniq Mapped Reads is the number and percentage of reads that mapped to a unique position in the reference genome in the clean reads; Multiple Mapped Reads is the number and percentage of reads that mapped to multiple positions in the reference genome in the clean reads. 
We followed stringent criteria and created a pipeline to identify lncRNAs, as shown in Figure 2A.

All mapped reads were assembled using Cufflinks. In addition, Cuffmerge was used to merge all the GTF files from the biological replicate and pooling samples to obtain a combined GTF file containing 146,397 transcripts. The information in the assembled transcripts also included the

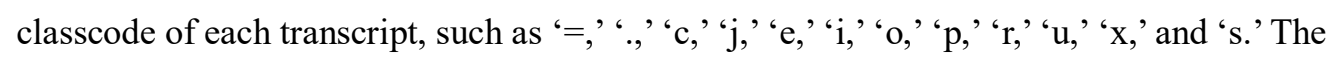
transcripts with non-coding potential classcodes of 'j, ' ‘, ' 'o,' ' $u$, ' ' $x$,' and '=' were reserved ${ }^{[35]}$, after which we filtered them with the length and exon numbers. Next, we predicted the coding potential by $\mathrm{CNCI}$ and $\mathrm{CPC}$ and compared the transcripts to the Pfam protein database. Finally, we identified 2,013 IncRNAs (Figure 2B). Among them, 334 were known transcripts and 1,679 were new transcripts, including 1,250 lincRNAs (62.10\%), 506 anti-sense lncRNAs (25.14\%), 154 sense lncRNAs (7.65\%), and 103 intronic lncRNAs (5.12\%) (Figure 2C). These 2,013 lncRNAs were distributed throughout all pig chromosomes, but chromosome 1 contained the most lncRNAs (Figure 2D). The majority of the lncRNA lengths were between 601 and 800 nt, and lncRNA with two exons were the most common, as shown in Figures 2E and F.

\section{Expression analysis of differentially and exclusively expressed IncRNAs and genes in} extremely-high or -low backfat pigs

Studies have shown that the expression level of lncRNAs is usually lower than that of protein-coding genes ${ }^{[36]}$. In the present study, we compared the expression of the putative IncRNAs with the mRNAs. We found that the lncRNAs tended to be expressed at a lower level than the protein-coding genes in both the biological replicate and pooling RNA-seq (Figures 3A 
and 4A). In the biological replicate RNA-seq, 1,512 genes and 220 lncRNAs (Additional File 2) were differentially expressed between the two groups, of which 820 genes were up-regulated in the $\mathrm{BH}$ group compared to those in the BL group, and 692 were down-regulated (Figures $3 \mathrm{~B}$ and D). The expression of 116 lncRNAs was up-regulated in the BH group, and 104 lncRNAs were down-regulated (Figure 3G). Next, we used the 1,512 and 220 differentially expressed mRNAs and lncRNAs, respectively, to perform a clustering analysis. As shown in the heatmap, the expression patterns of the samples in the $\mathrm{BH}$ group were distinguished from their expression patterns in the BL group, both for the DEGs (Figure 3E) and DELs (Figure 3H).

In the pooling RNA-seq, we carried out the same analysis as that used for the biological replicate RNA-seq. We found that 2,240 genes and 127 lncRNAs (Additional File 2) were differentially expressed in the two groups, of which 1,206 genes were up-regulated in the HIGH group compared to those in the LOW group, and 1,034 were down-regulated (Figures $4 \mathrm{~B}$ and E). There were 83 lncRNAs with up-regulated expression levels in the HIGH group, and 44 lncRNAs were down-regulated (Figure 4F).

In addition, to further explore the difference in gene expression between the high and low backfat thickness groups, we analyzed the lncRNAs and genes exclusively expressed in the two groups. There were 620 genes and 133 lncRNAs exclusively expressed in the BH group and 607 genes and 118 lncRNAs exclusively expressed in the BL group (Figures 3C and F). Moreover, based on the pooling RNA-seq results, we identified 643 genes and 136 lncRNAs exclusively expressed in the HIGH group and 892 genes and 177 lncRNAs exclusively expressed in the LOW group (Figures 4C and D). 
GO and KEGG functional enrichment analysis of DEGs

47

We used the differentially expressed genes detected in the two sequencing methods to perform a functional enrichment analysis. Figures $5 \mathrm{~A}$ and $\mathrm{C}$ show the ten most significant $(\mathrm{FDR}<0.05)$ terms in the Biological Process (BP) class and the five most significant terms in the Cellular Component (CC) and Molecular Function (MF) classes. In the biological replicate RNA-seq, a total of 977 DEGs were annotated in GO terms. We found many terms directly related to fat development and metabolism, especially in the BP class, including 'tricarboxylic acid cycle,' 'triglyceride homeostasis,' and 'triglyceride catabolic process.' Furthermore, there were four mRNAs enriched to 'positive regulation of triglyceride catabolic process'. This GO term was also significant but not within the top ten; so, it is not displayed in Figure 5. In addition, DEGs were also enriched in some glucose metabolism pathways such as 'gluconeogenesis' and 'glycolytic process.' The results of the pooling RNA-seq are shown in Figure 5C. We focused on several terms, including 'positive regulation of fatty acid biosynthetic process,' 'cholesterol biosynthetic process,' 'lipoprotein metabolic process,' and 'carbohydrate metabolic process.' We also found terms such as 'gluconeogenesis' and 'glycolytic process,' which were the same as those in the biological replicate RNA-seq. All the terms that were considered important are highlighted by a red frame in Figure 5.

Meanwhile, these DEGs also underwent a KEGG analysis to annotate their functions. Figures 5B and D show the most significant 20 pathways in the KEGG results. In the biological replicate RNA-seq, the pathways related to glucose and lipoid metabolism, including 'Glycolysis / Gluconeogenesis,' 'Citrate cycle (TCA cycle),' 'Pyruvate metabolism,' 'Fat digestion and 
absorption,' and 'Starch and sucrose metabolism,' were significantly enriched. Moreover, in the pooling RNA-seq, we also found two new pathways that were not previously enriched, 'Synthesis and degradation of ketone bodies' and 'Pentose phosphate pathway,' both of which are important in the glucose metabolism program. We also circled the important pathways with a red frame in Figure 5.

We used the exclusively expressed genes from the biological replicate RNA-seq to perform the functional enrichment analysis. However, there are only a few KEGG pathways related to glucose and lipoid metabolism, most of which involved the exclusively expressed genes in the BH group. These pathways include 'Fat digestion and absorption,' 'Synthesis and degradation of ketone bodies,' and 'Pentose phosphate pathway.' Therefore, we believe that the other GO terms and KEGG pathways are not as necessary. These results are shown in Additional File 1.

\section{Target gene prediction of differentially expressed IncRNAs}

To further understand the potential function of IncRNA in fat deposition, we predicted the target genes of the lncRNAs. The general method of predicting lncRNA target genes is to search upstream or downstream to identify nearby protein-coding regions, termed as cis-regulating target genes ${ }^{[37]}$. It has been reported that lncRNA can regulate coding genes around 10 to $500 \mathrm{~kb}$ up and downstream ${ }^{[38]}$. We searched the nearby coding genes around $100 \mathrm{~kb}$ up and downstream from the 39 DELs, which were common in the two sequencing results. We found 76 pairs of cis-regulatory relationships between 39 DELs and 67 genes (Additional File 3).

If the expression patterns of lncRNA and mRNA show a highly positive or negative correlation, their functions may be highly correlated ${ }^{[39]}$. Therefore, we conducted a Spearman's rank 
correlation analysis between the expression of the DELs and DEGs. The expression levels of 116

DEGs were significantly correlated with the 39 DELs (correlation coefficient $>0.934, P<0.05$ ).

They were also considered as potential target genes of these DELs. We used 39 DELs and their target genes to draw a picture of the regulatory network to investigate the relationship between them (Figure 6). We highlighted the lncRNAs and their target genes for further analysis with yellow nodes and red labels.

\section{Comparison of biological replicate and pooling RNA-seq}

We utilized fragments per kilobase of transcript per million mapped reads (FPKM) $\geqslant 0.1$ as the criteria to ensure expression in all samples, then tested the expression levels of the lncRNAs and genes in each sample using biological replicate and pooling RNA-seq. A total of 1,604 lncRNAs and 16,002 genes were identified in both sequencing results. We also found 266 unique lncRNAs and 2,505 unique genes in the biological replicate RNA-seq; however, only 14 lncRNAs and 1,124 genes were uniquely detected in the pooling RNA-seq (Figures 7A and B). Next, GO and KEGG functional enrichment analysis were performed again with the unique genes of the two sequencing methods. As shown in Figures 7C-F, none of the GO terms or KEGG pathways were related to glucose and lipid metabolism in this analysis.

\section{Discussion}

In our study, six full-sib Landrace pigs with either extremely-high or -low backfat thickness were taken as paired samples, and their back subcutaneous tissues were collected. We compared the transcriptome data of the two groups using two methods of RNA-seq, identified the differentially 
111

112

113

expressed lncRNAs and genes, then analyzed their function in fat development. The results of our study provide a basis for the selection of pigs for breeding using lncRNAs and their target genes to improve the fat deposition traits of pigs.

In this study, we identified a total of 19,631 genes and 2,013 lncRNAs. In accordance with the results of a previous study ${ }^{[36]}$, we found that the expression of lncRNA was much lower than that of mRNA. Our results are similar to those in several previously published articles on adipose tissue transcriptome sequencing. For example, Miao identified 4,910 lncRNAs, 119 of which were differentially expressed in the intramuscular fat tissue of Jinhua and Changbai pigs ${ }^{[40]}$. The results for the functional enrichment of the DEGs showed that some genes were enriched to pathways related to glucose and lipid metabolism. In addition, these pathways were confirmed to be related to lipid metabolism in previous studies, including $A P O A 1^{[41]}$ and $S T A R D 3^{[42]}$. Chen identified 581 putative lincRNAs related to pig muscle growth and fat deposition, and their target genes were involved in fat deposition-related processes such as the lipid metabolic process and fatty acid degradation ${ }^{[43]}$. The KEGG results showed that the meaningful pathways were mostly concentrated on glucose metabolism. Some of these pathways, such as 'Glycolysis /

Gluconeogenesis,' were also found in a previous study by our team ${ }^{[44]}$. Although they are not directly related to lipid synthesis and metabolism, glucose and lipids can change into each other and participate together in the tricarboxylic acid cycle. Therefore, the process of glucose metabolism can have an indirect effect on lipid metabolism.

As a kind of non-coding RNA, the main role of lncRNA is to regulate their target genes: cis-regulating nearby protein-coding genes and trans-regulating distant protein-coding genes. We determined 67 cis-target genes and 116 trans-target genes regulated by DELs. The two target 
133

genes we focused on were $A C A C B$ and $A C S L 3$, both of which have been found to be related to lipid metabolism in other studies. ACLS3 (acyl-CoA synthetase long chain family member 3) is located $64 \mathrm{~kb}$ downstream of TCONS-00052400. At the beginning of fatty acid metabolism, long-chain acyl-CoA synthetase (ACSL) can activate the conversion of long-chain fatty acids to fatty acyl-CoA ${ }^{[45]}$. The ACSL family contains five different isoforms, including ACSL3, which play different roles in lipid metabolism. Some previous studies showed that knockdown of ACSL3 may significantly reduce the activity of several lipid-producing transcription factors like peroxisome proliferator-activated receptor- $\gamma$ and sugar response element binding protein, and then regulate the fat production process in the liver ${ }^{[46]}$. Furthermore, ACSL3 can also affect the secretion of very low-density lipoproteins (VLDL) by promoting the synthesis of lecithin ${ }^{[47]}$. $A C A C B$ (acetyl-CoA carboxylase beta) is a differentially expressed gene that is cis-regulated by the lncRNA TCONS-00041740, which is located on the antisense strand of $A C A C B$. Moreover, in the KEGG enrichment analysis, $A C A C B$ was involved in the 'pyruvate metabolism' pathway. Based on previous studies, we knew that $A C A C B$ is the rate-limiting enzyme in fatty acid oxidation $^{[48]}$. Moreover, in $A C A C B$ knockout mice, continuous fatty acid oxidation increases insulin sensitivity, and feeding them a high fat/high carbohydrate diet is more likely to cause obesity and diabetes ${ }^{[49]}$. In a previous study by $\mathrm{Li}, A C A C B$ was shown to be a marker gene for childhood obesity ${ }^{[50]}$.

Some genes were exclusively expressed in one of the two groups. For example, PLCB2

(phospholipase $\mathrm{C}$ beta 2) was exclusively expressed in the BL group. Phospholipase $\mathrm{C}$ is a class of glycerol phospholipid hydrolases, hydrolyzing the glycerol phosphate C3 site ${ }^{[51]}$. The protein encoded by $P L C B 2$ is a phosphodiesterase that catalyzes the hydrolysis of phosphatidylinositol 4, 
155

156

157

5-bisphosphate to the secondary messengers inositol 1,4,5-trisphosphate (IP3) and diacylglycerol.

In addition, the gene PLA2G12B was exclusively expressed in the BH group. PLA2G12B

(phospholipase A2 group XIIB) is encoded by this gene and belongs to the phospholipase A2

(PLA2) group of enzymes, which plays a role in lipid hydrolysis by releasing free fatty acids and

lysophospholipids ${ }^{[52]}$. Studies have shown that a reduction of $P L A 2 G 12 B$ decreases the amount of serum triglyceride (TG)-rich VLDL particles secreted by the liver, resulting in a reduction in TG content $^{[53]}$. PLA2G12B can also participate in the pathogenesis of idiopathic membranous nephropathy (iMN) by regulating lipid metabolism ${ }^{[54]}$. In a previous study by Guan,

PLA2G12B-null mice had obvious accumulation of large lipid droplets in the liver, displaying the fatty liver phenotype ${ }^{[55]}$. These results indicate that the genes exclusively expressed in the high or low backfat groups may also have a certain regulatory effect on lipid metabolism.

Biological replicate and pooling RNA-seq are two conventional methods used in RNA-seq experiments. In this study, biological replicate RNA-seq detected more lncRNAs and genes than did pooling RNA-seq. Few unique lncRNAs were identified in the latter. However, in the function annotation, the unique genes of both sequencing methods did not enrich the GO terms and KEGG pathways related to glucose and lipid metabolism. To a certain extent, this indicates that the unique genes have no direct influence on fat deposition in Landrace pigs. These results illustrate that when RNA-seq is used to excavate key genes, biological replicate RNA-seq is superior in 'quantity', but there is no obvious 'qualitative' difference between the two methods. We propose that when the experimental conditions are constrained, such as if the cost of sequencing is too high or the samples are difficult to obtain, replacing biological replicate RNA-seq with pooling RNA-seq can also achieve important results. However, using RNA-seq to determine important 
177

lncRNAs, microRNAs, or other noncoding RNAS via pooling RNA-seq only may cause some important information to be masked.

During pig growth, the excessive development of adipose tissue leads to an excessive accumulation of lipids, which affects the carcass quality of pigs. Overweight pigs show low lean meat rates, low feed conversion rates, and slow growth ${ }^{[56]}$, while overweight sows may experience dystocia and postpartum disease ${ }^{[57]}$. Numerous studies have shown that pigs have many similarities with humans in anatomical structure, physiological metabolism, and disease mechanisms ${ }^{[58,59]}$. Therefore, pigs have many advantages as an animal model for human disease research, especially related to obesity ${ }^{[60]}$. For example, after feeding China Guizhou miniature pigs a high-fat, high-sugar diet for 6 months, $\mathrm{Xi}^{[61]}$ found that the pigs developed insulin resistance, mild diabetes, and atherosclerotic lesions. Therefore, this model pig may be a suitable animal model for studying diabetic dyslipidemia complicated with atherosclerosis. Pig backfat thickness can directly reflect their body fat content. Our study obtained differentially expressed lncRNAs between pigs with extremely-high and -low backfat thickness, providing a novel approach for the use of lncRNAs and their target genes to screen low backfat pigs in future breeding work, thereby further improving fat deposition traits in pigs.

\section{Conclusions}

In this study, we identified a total of 307 DELs and 3,034 DEGs between pigs with extremely-high and -low fat deposition traits. After the analysis of potential cis- and trans- target genes, we found 183 genes that could be regulated by DELs. Through further functional analysis, we found that two potential targeting relationships may have an important regulatory effect on fat deposition: 
199

200

201

202

203

204

205

206

207

208

209

210

211

212

213

TCONS-00041740 to ACACB, and TCONS-00052400 to ACSL3. These results can provide useful information for understanding the regulation of fat deposition by lncRNA in pigs. However, further genetic experiments are still needed to validate the association of the IncRNA and mRNA functions presented in this study. 
222 Competing Interests: The authors declare no competing interests.

Funding: This research was financially supported by the National Key R\&D Program of China

Program General Project (KM201910020010).

Authors' contributions: Y.L., Y.Y., and C.W. designed the study. Y.L., K.X., F.Z., X.Z., and H.L.

K.X. wrote the paper. Y.Y. and C.W. revised the manuscript. All authors read and approved the final manuscript.

Acknowledgments: Grateful to the molecular quantitative genetics team of China Agricultural

University for their guidance. Authors are appreciated to worker from Tianjin Ninghe primary pig 
243

244

245

246

247

248

249

250

251

252

253

254

255

256

257

258

259

260

261

262

263

264

\section{References:}

[1] Tummaruk P, Lundeheim N, Einarsson S, Dalin AM. Effect of birth litter size, birth parity number, growth rate, backfat thickness and age at first mating of gilts on their reproductive performance as sows. Anim Reprod Sci. 2001;66(3-4):225-237.

doi:10.1016/s0378-4320(01)00095-1

[2] Zhang J, Cui L, Ma J, Chen C, Yang B, Huang L. Transcriptome analyses reveal genes and pathways associated with fatty acid composition traits in pigs. Anim Genet. 2017;48(6):645-652. doi:10.1111/age.12597

[3] Zhao W, Mu Y, Ma L, et al. Systematic identification and characterization of long intergenic non-coding RNAs in fetal porcine skeletal muscle development. Sci Rep. 2015;5:8957. Published 2015 Mar 10. doi:10.1038/srep08957

[4] Hajer GR, van Haeften TW, Visseren FL. Adipose tissue dysfunction in obesity, diabetes, and vascular diseases. Eur Heart J. 2008;29(24):2959-2971. doi:10.1093/eurheartj/ehn387

[5] Willer CJ, Speliotes EK, Loos RJ, et al. Six new loci associated with body mass index highlight a neuronal influence on body weight regulation. Nat Genet. 2009;41(1):25-34. doi:10.1038/ng.287

[6] Kapranov P, Cheng J, Dike S, et al. RNA maps reveal new RNA classes and a possible function for pervasive transcription. Science. 2007;316(5830):1484-1488.

doi:10.1126/science. 1138341

[7] Ørom UA, Derrien T, Beringer M, et al. Long noncoding RNAs with enhancer-like function in human cells. Cell. 2010;143(1):46-58. doi:10.1016/j.cell.2010.09.001

[8] Yoon JH, Abdelmohsen K, Srikantan S, et al. LincRNA-p21 suppresses target mRNA 
translation [published correction appears in Mol Cell. 2013 Apr 25;50(2):303]. Mol Cell. 2012;47(4):648-655. doi:10.1016/j.molcel.2012.06.027

[9] Vennin C, Spruyt N, Robin YM, Chassat T, Le Bourhis X, Adriaenssens E. The long non-coding RNA $91 \mathrm{H}$ increases aggressive phenotype of breast cancer cells and up-regulates H19/IGF2 expression through epigenetic modifications. Cancer Lett. 2017;385:198-206. doi:10.1016/j.canlet.2016.10.023

[10] Alvarez-Dominguez JR, Bai Z, Xu D, et al. De Novo Reconstruction of Adipose Tissue Transcriptomes Reveals Long Non-coding RNA Regulators of Brown Adipocyte Development [published correction appears in Cell Metab. 2015 Jun 2;21(6):918]. Cell Metab. 2015;21(5):764-776. doi:10.1016/j.cmet.2015.04.003

[11] Li M, Sun X, Cai H, et al. Long non-coding RNA ADNCR suppresses adipogenic differentiation by targeting miR-204. Biochim Biophys Acta. 2016;1859(7):871-882. doi:10.1016/j.bbagrm.2016.05.003

[12] Xing K, Zhao X, Ao H, et al. Transcriptome analysis of miRNA and mRNA in the livers of pigs with highly diverged backfat thickness. Sci Rep. 2019;9(1):16740. Published 2019 Nov 14. doi:10.1038/s41598-019-53377-x

[13] Xing K, Zhu F, Zhai L, et al. Identification of genes for controlling swine adipose deposition by integrating transcriptome, whole-genome resequencing, and quantitative trait loci data. Sci Rep. 2016;6:23219. Published 2016 Mar 21. doi:10.1038/srep23219

[14] Bolger AM, Lohse M, Usadel B. Trimmomatic: a flexible trimmer for Illumina sequence data. Bioinformatics. 2014;30(15):2114-2120. doi:10.1093/bioinformatics/btu170

[15] Andrews, S. FastQC: A Quality Control Tool for High Throughput Sequence Data, version 
[16] Dai M, Thompson RC, Maher C, et al. NGSQC: cross-platform quality analysis pipeline for deep sequencing data. BMC Genomics. 2010;11 Suppl 4(Suppl 4):S7. Published 2010 Dec 2. doi:10.1186/1471-2164-11-S4-S7

[17] Langmead B, Salzberg SL. Fast gapped-read alignment with Bowtie 2. Nat Methods. 2012;9(4):357-359. Published 2012 Mar 4. doi:10.1038/nmeth.1923

[18] Kim D, Pertea G, Trapnell C, Pimentel H, Kelley R, Salzberg SL. TopHat2: accurate alignment of transcriptomes in the presence of insertions, deletions and gene fusions. Genome Biol. 2013;14(4):R36. Published 2013 Apr 25. doi:10.1186/gb-2013-14-4-r36

[19] Trapnell C, Roberts A, Goff L, et al. Differential gene and transcript expression analysis of RNA-seq experiments with TopHat and Cufflinks [published correction appears in Nat Protoc. 2014 Oct;9(10):2513]. Nat Protoc. 2012;7(3):562-578. Published 2012 Mar 1. doi:10.1038/nprot.2012.016

[20] Kong L, Zhang Y, Ye ZQ, et al. CPC: assess the protein-coding potential of transcripts using sequence features and support vector machine. Nucleic Acids Res. 2007;35(Web Server issue):W345-W349. doi:10.1093/nar/gkm391

[21] Sun L, Luo H, Bu D, et al. Utilizing sequence intrinsic composition to classify protein-coding and long non-coding transcripts. Nucleic Acids Res. 2013;41(17):e166. doi:10.1093/nar/gkt646 [22] Finn RD, Coggill P, Eberhardt RY, et al. The Pfam protein families database: towards a more sustainable future. Nucleic Acids Res. 2016;44(D1):D279-D285. doi:10.1093/nar/gkv1344

[23] Chen G, Cheng X, Shi G, et al. Transcriptome Analysis Reveals the Effect of Long Intergenic Noncoding RNAs on Pig Muscle Growth and Fat Deposition. Biomed Res Int. 
2019;2019:2951427. Published 2019 Jun 25. doi:10.1155/2019/2951427

[24] Anders S, Pyl PT, Huber W. HTSeq--a Python framework to work with high-throughput sequencing data. Bioinformatics. 2015;31(2):166-169. doi:10.1093/bioinformatics/btu638

[25] Robinson MD, McCarthy DJ, Smyth GK. edgeR: a Bioconductor package for differential expression analysis of digital gene expression data. Bioinformatics. 2010;26(1):139-140. doi:10.1093/bioinformatics/btp616

[26] Raivo Kolde. Pheatmap: Pretty Heatmaps. R Package Version 1.012. 2019.

[27] Ashburner M, Ball CA, Blake JA, et al. Gene ontology: tool for the unification of biology.

The Gene Ontology Consortium. Nat Genet. 2000;25(1):25-29. doi:10.1038/75556

[28] Ogata H, Goto S, Sato K, Fujibuchi W, Bono H, Kanehisa M. KEGG: Kyoto Encyclopedia of Genes and Genomes. Nucleic Acids Res. 1999;27(1):29-34. doi:10.1093/nar/27.1.29

[29] Dennis G Jr, Sherman BT, Hosack DA, et al. DAVID: Database for Annotation, Visualization, and Integrated Discovery. Genome Biol. 2003;4(5):P3.

[30] Xie C, Mao X, Huang J, et al. KOBAS 2.0: a web server for annotation and identification of enriched pathways and diseases. Nucleic Acids Res. 2011;39(Web Server issue):W316-W322. doi:10.1093/nar/gkr483

[31] Wickham H. Ggplot2: Elegant Graphics for Data Analysis. German: Springer. 2009.

[32] Zhan S, Dong Y, Zhao W, et al. Genome-wide identification and characterization of long non-coding RNAs in developmental skeletal muscle of fetal goat. BMC Genomics. 2016;17(1):666. Published 2016 Aug 22. doi:10.1186/s12864-016-3009-3

[33] Hou X, Du Y, Liu X, et al. Genome-Wide Analysis of Long Non-Coding RNAs in Potato and Their Potential Role in Tuber Sprouting Process. Int J Mol Sci. 2017;19(1):101. Published 2017 
Dec 29. doi:10.3390/ijms19010101

[34] Quinlan AR, Hall IM. BEDTools: a flexible suite of utilities for comparing genomic features. Bioinformatics. 2010;26(6):841-842. doi:10.1093/bioinformatics/btq033

[35] Lv J, Cui W, Liu H, et al. Identification and characterization of long non-coding RNAs related to mouse embryonic brain development from available transcriptomic data. PLoS One. 2013;8(8):e71152. Published 2013 Aug 14. doi:10.1371/journal.pone.0071152

[36] Liu S, Wang Z, Chen D, et al. Annotation and cluster analysis of spatiotemporal- and sex-related lncRNA expression in rhesus macaque brain. Genome Res. 2017;27(9):1608-1620. doi:10.1101/gr.217463.116

[37] Zhang Q, Chao TC, Patil VS, et al. The long noncoding RNA ROCKI regulates inflammatory gene expression. EMBO J. 2019;38(8):e100041. doi:10.15252/embj.2018100041

[38] Bonasio R, Shiekhattar R. Regulation of transcription by long noncoding RNAs. Annu Rev Genet. 2014;48:433-455. doi:10.1146/annurev-genet-120213-092323

[39] Atala A. Re: IncRNA-dependent mechanisms of androgen-receptor-regulated gene activation programs. J Urol. 2014;191(5):1470-1471. doi:10.1016/j.juro.2014.02.011

[40] Miao Z, Wang S, Zhang J, et al. Identification and comparison of long non-conding RNA in Jinhua and Landrace pigs. Biochem Biophys Res Commun. 2018;506(3):765-771. doi:10.1016/j.bbrc.2018.06.028

[41] Xepapadaki E, Maulucci G, Constantinou C, et al. Impact of apolipoprotein A1 - or lecithin:cholesterol acyltransferase-deficiency on white adipose tissue metabolic activity and glucose homeostasis in mice. Biochim Biophys Acta Mol Basis Dis. 2019;1865(6):1351-1360. doi:10.1016/j.bbadis.2019.02.003 
353

354

355

356

357

358

359

360

361

362

363

364

365

366

367

368

369

370

371

372

373

374

[42] Zhou X, Gao H, Guo Y, Chen Y, Ruan XZ. Knocking down Stard3 decreases adipogenesis

with decreased mitochondrial ROS in 3T3-L1 cells. Biochem Biophys Res Commun.

2018;504(2):387-392. doi:10.1016/j.bbrc.2018.06.030

[43] Chen G, Cheng X, Shi G, et al. Transcriptome Analysis Reveals the Effect of Long Intergenic

Noncoding RNAs on Pig Muscle Growth and Fat Deposition. Biomed Res Int.

2019;2019:2951427. Published 2019 Jun 25. doi:10.1155/2019/2951427

[44] Xing K, Wang K, Ao H, et al. Comparative adipose transcriptome analysis digs out genes

related to fat deposition in two pig breeds. Sci Rep. 2019;9(1):12925. Published 2019 Sep 9.

doi:10.1038/s41598-019-49548-5

[45] Mashek DG, Li LO, Coleman RA. Long-chain acyl-CoA synthetases and fatty acid

channeling. Future Lipidol. 2007;2(4):465-476. doi:10.2217/17460875.2.4.465

[46] Bu SY, Mashek MT, Mashek DG. Suppression of long chain acyl-CoA synthetase 3 decreases hepatic de novo fatty acid synthesis through decreased transcriptional activity. J Biol Chem.

2009;284(44):30474-30483. doi:10.1074/jbc.M109.036665

[47] Yao H, Ye J. Long chain acyl-CoA synthetase 3-mediated phosphatidylcholine synthesis is required for assembly of very low density lipoproteins in human hepatoma Huh7 cells. J Biol Chem. 2008;283(2):849-854. doi:10.1074/jbc.M706160200

[48] Abu-Elheiga L, Matzuk MM, Abo-Hashema KA, Wakil SJ. Continuous fatty acid oxidation and reduced fat storage in mice lacking acetyl-CoA carboxylase 2. Science.

2001;291(5513):2613-2616. doi:10.1126/science.1056843

[49] Abu-Elheiga L, Oh W, Kordari P, Wakil SJ. Acetyl-CoA carboxylase 2 mutant mice are protected against obesity and diabetes induced by high-fat/high-carbohydrate diets. Proc Natl 
Acad Sci U S A. 2003;100(18):10207-10212. doi:10.1073/pnas.1733877100

[50] Li L, Wang G, Li N, Yu H, Si J, Wang J. Identification of key genes and pathways associated with obesity in children. Exp Ther Med. 2017;14(2):1065-1073. doi:10.3892/etm.2017.4597

[51] Kanematsu T, Oue K, Okumura T, et al. Phospholipase C-related catalytically inactive protein: A novel signaling molecule for modulating fat metabolism and energy expenditure. J Oral Biosci. 2019;61(2):65-72. doi:10.1016/j.job.2019.04.002

[52] Jackisch L, Kumsaiyai W, Moore JD, et al. Differential expression of Lp-PLA2 in obesity and type 2 diabetes and the influence of lipids. Diabetologia. 2018;61(5):1155-1166. doi:10.1007/s00125-018-4558-6

[53] Liu Q, Yang M, Fu X, et al. Activation of farnesoid X receptor promotes triglycerides lowering by suppressing phospholipase A2 G12B expression. Mol Cell Endocrinol. 2016;436:93-101. doi:10.1016/j.mce.2016.07.027

[54] Wu D, Yu Z, Zhao S, Qu Z, Sun W, Jiang Y. Lipid metabolism participates in human membranous nephropathy identified by whole-genome gene expression profiling. Clin Sci (Lond). 2019;133(11):1255-1269. Published 2019 Jun 13. doi:10.1042/CS20181110

[55] Guan M, Qu L, Tan W, Chen L, Wong CW. Hepatocyte nuclear factor-4 alpha regulates liver triglyceride metabolism in part through secreted phospholipase $\mathrm{A}_{2}$ GXIIB. Hepatology. 2011;53(2):458-466. doi:10.1002/hep.24066

[56] Rocha D, Plastow G. Commercial pigs: an untapped resource for human obesity research?. Drug Discov Today. 2006;11(11-12):475-477. doi:10.1016/j.drudis.2006.04.009 [57] Xu M, Che L, Yang Z, et al. Effect of High Fat Dietary Intake during Maternal Gestation on Offspring Ovarian Health in a Pig Model. Nutrients. 2016;8(8):498. Published 2016 Aug 13. 
397

398

399

400

401

402

403

404

405

406

407

408

409

410

411

412

413

414

415

416

417

418

419

doi:10.3390/nu8080498

[58] Fang X, Mou Y, Huang Z, et al. The sequence and analysis of a Chinese pig

genome. Gigascience. 2012;1(1):16. Published 2012 Nov 15. doi:10.1186/2047-217X-1-16

[59] Murakami T, Hitomi S, Ohtsuka A, Taguchi T, Fujita T. Pancreatic insulo-acinar portal systems in humans, rats, and some other mammals: scanning electron microscopy of vascular casts. Microsc Res Tech. 1997;37(5-6):478-488.

doi:10.1002/(SICI)1097-0029(19970601)37:5/6<478::AID-JEMT10>3.0.CO;2-N

[60] Bellinger DA, Merricks EP, Nichols TC. Swine models of type 2 diabetes mellitus: insulin resistance, glucose tolerance, and cardiovascular complications. ILAR J. 2006;47(3):243-258. doi:10.1093/ilar.47.3.243.

[61] Xi S, Yin W, Wang Z, et al. A minipig model of high-fat/high-sucrose diet-induced diabetes and atherosclerosis. Int J Exp Pathol. 2004;85(4):223-231. doi:10.1111/j.0959-9673.2004.00394.x

\section{Figure Captions}

Figure 1 Technical route of the current study

\section{Figure 2 Summary of putative long non-coding RNA (IncRNA) characteristics in Landrace}

\section{pig backfat}

(A) Identification pipeline of lncRNAs; (B) Venn diagram of identified lncRNAs from the Coding Potential Calculator (CPC), Coding-Non-Coding Index (CNCI), and Pfam database; (C) Type and 
number of putative IncRNAs; (D) Chromosome distribution of lncRNAs; (E) Length distribution of lncRNAs; (F) Exon number distribution of lncRNAs.

Figure 3 Expression of long non-coding RNAs (IncRNAs) and genes in biological replicate RNA sequencing (RNA-seq) in extremely-high and -low backfat pigs

(A) Comparison of the expression levels between the lncRNAs and messenger RNAs in six samples. The value of $\log 10$ (Fragments per kilobase of transcript per million mapped reads $[\mathrm{FPKM}]+1$ ) is plotted on the Y-axis; (B) Number of differentially expressed lncRNAs (DELs) and differentially expressed genes (DEGs) between the high and low groups; (C) Venn diagram of genes from each group; (D) Volcano plot of DEGs. The Y-axis is the value of - $\log 10$ (false discover rate[FDR]) and the $\mathrm{X}$-axis is the value of $\log 2(\mathrm{FC})$. The two threshold lines show the standard of $\mathrm{FDR}=0.05$ and $\mathrm{FC}=2$; (E) Heatmap of genes in six samples. The color scale indicates the FPKM values. Orange indicates high expression and purple indicates low expression; (F) Venn diagram of IncRNAs from each group; (G) Volcano plot of DELs; (H) Heatmap of IncRNAs in six samples.

Figure 4 Expression of long non-coding RNAs (IncRNAs) and genes based on the pooling RNA sequencing results for extremely-high and -low backfat pigs

(A) Comparison of expression levels between the lncRNAs and messenger RNAs in two groups;

(B) Number of differentially expressed lncRNAs (DELs) and differentially expressed genes (DEGs) between the HIGH and LOW groups; (C) Venn diagram of the genes from each group; (D) Venn diagram of the lncRNAs from each group; (E) Volcano plot of the DEGs; (F) Volcano plot of the DELs. 
443

444

445

446

447

448

449

450

451

452

453

454

455

456

457

458

459

460

461

462

463

Figure 5 Functional enrichment analysis of differentially expressed genes (DEGs)

(A) Gene Ontology (GO) annotation of DEGs in biological replicate RNA sequencing (RNA-seq);

(B) Kyoto Encyclopedia of Genes and Genomes (KEGG) pathway analysis of DEGs in biological replicate RNA-seq; (C) GO annotation of DEGs in pooling RNA-seq; (D) KEGG pathway analysis of DEGs in pooling RNA-seq. The red boxes indicate the GO terms and KEGG pathways related to glucose and lipoid metabolism.

\section{Figure 6 Network of regulatory relationships between differentially expressed long}

non-coding RNAs (DELs) and their target genes

DELs are indicated in red ovals; target genes are indicated in blue diamonds; the important long non-coding RNAS and genes are highlighted with yellow nodes and red labels.

Figure 7 Analysis of unique genes in biological replicate and pooling RNA sequencing (RNA-seq)

(A) Venn diagram of unique genes in two sequencing methods; (B) Venn diagram of unique long non-coding RNAS (lncRNAs) in two sequencing methods; (C) Gene Ontology (GO) annotation of unique genes in biological replicate RNA-seq; (D) Kyoto Encyclopedia of Genes and Genomes (KEGG) pathway analysis of unique genes in biological replicate RNA-seq; (E) GO annotation of unique genes in pooling RNA-seq; (F) KEGG pathway analysis of unique genes in pooling RNA-seq. 
Figures

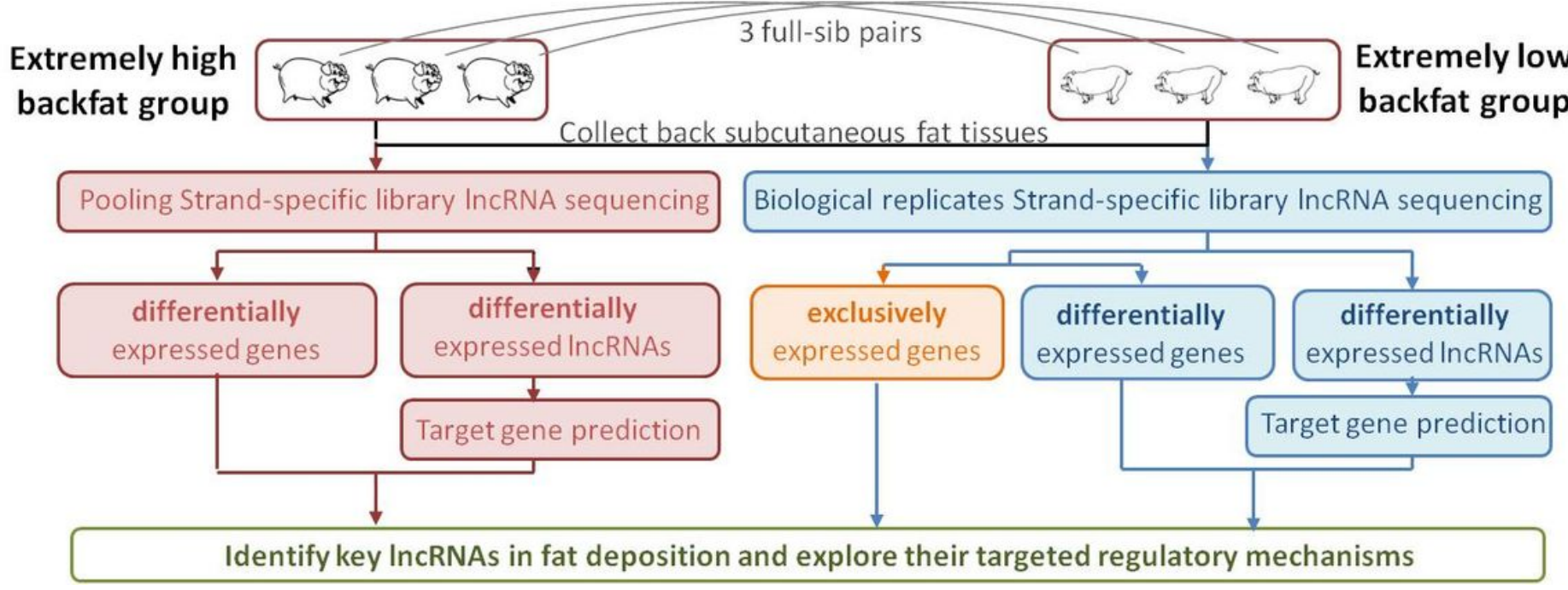

\section{Figure 1}

Figure 1 
A

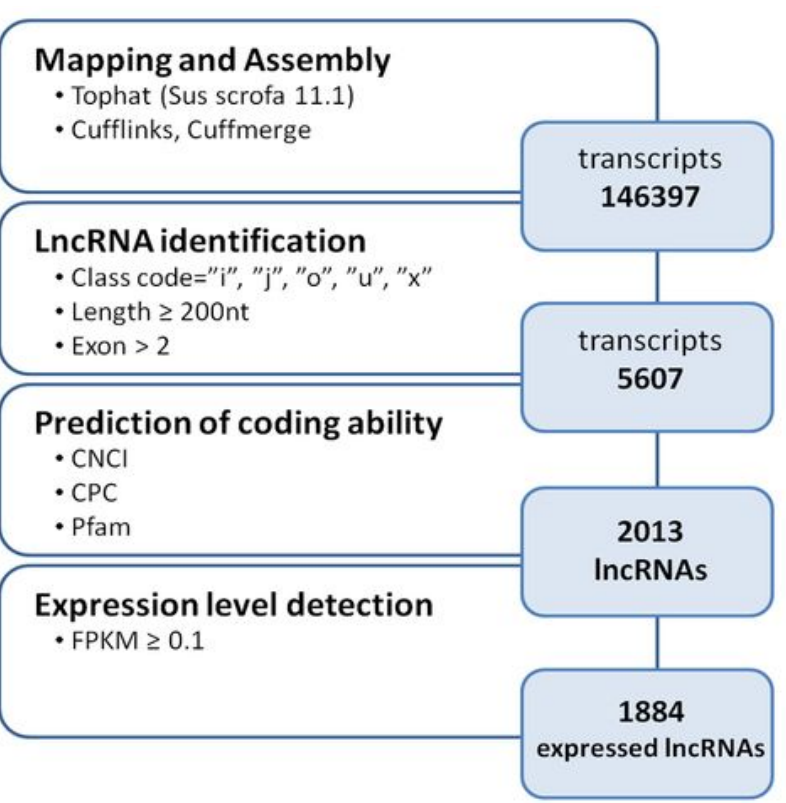

B

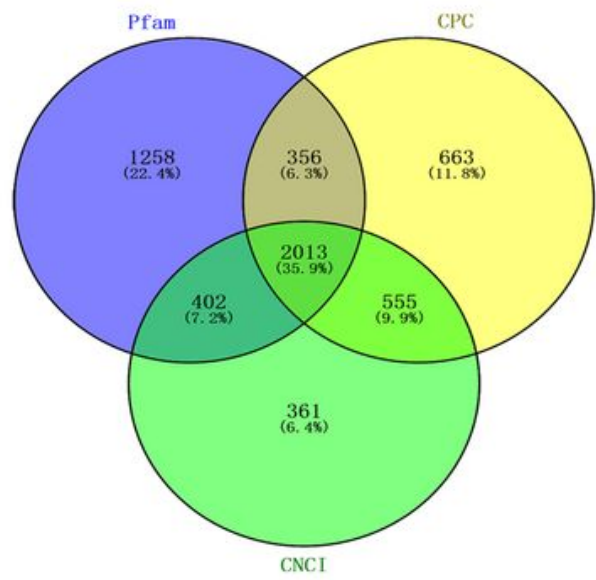

C

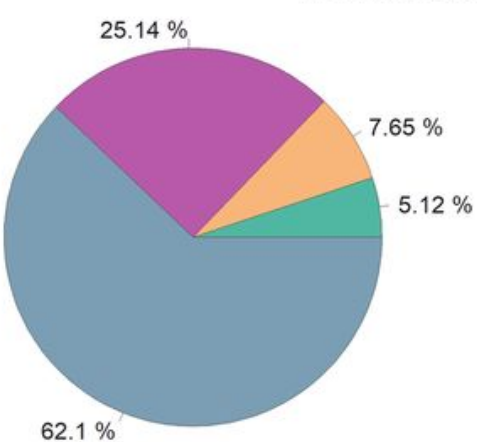

Intronic IncRNA

Sense IncRNA

Anti-senselncRNA

Intergenic IncRNA

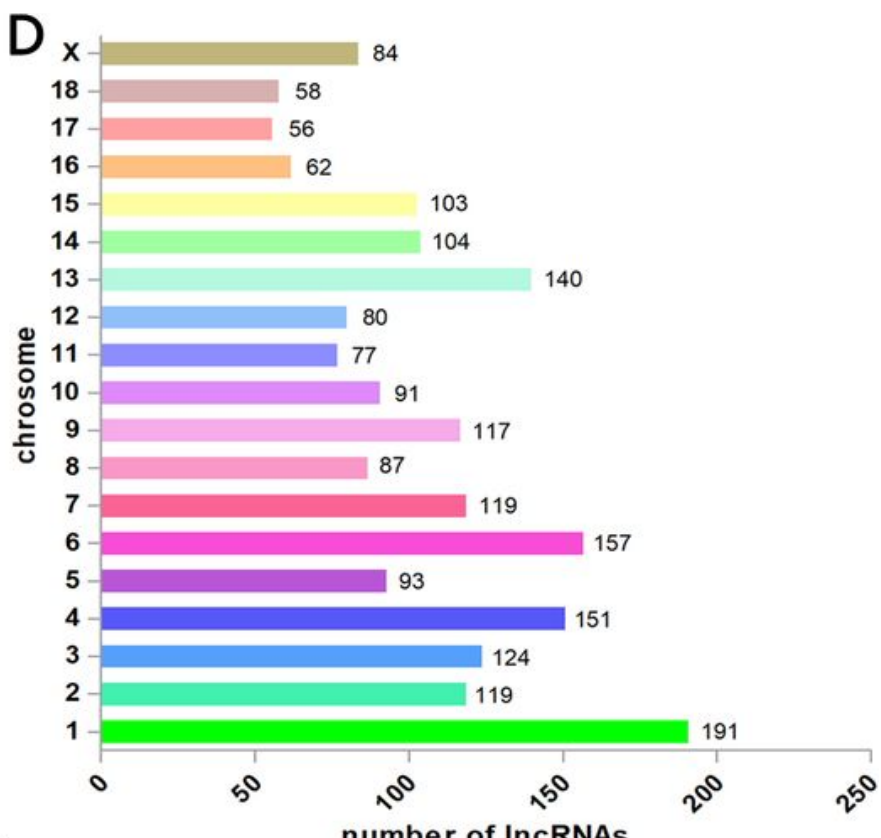

E 400
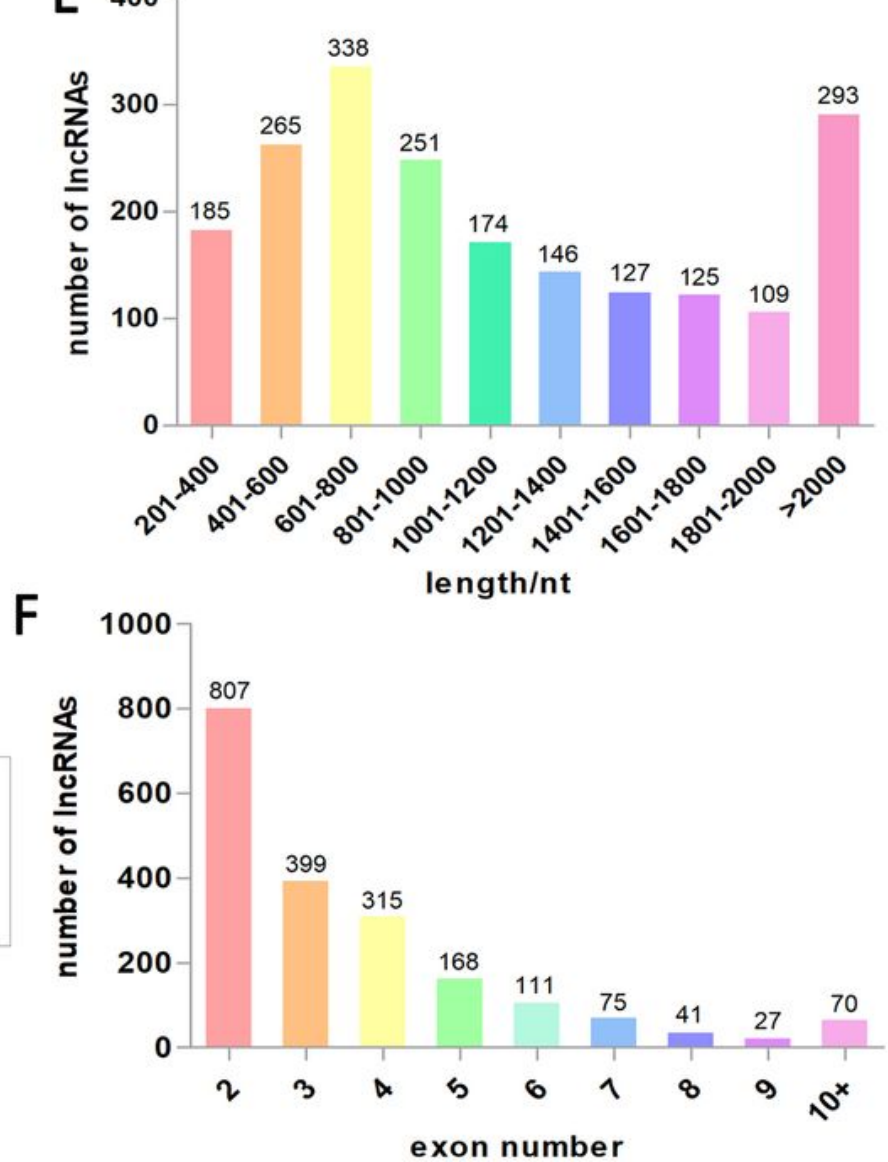

exon number

Figure 2

Figure 2 
A

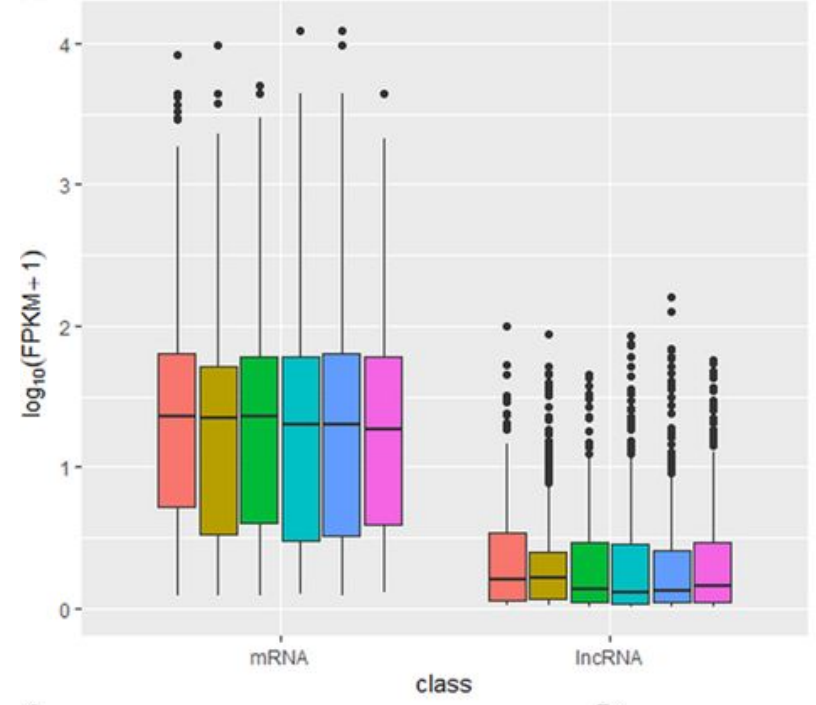

C

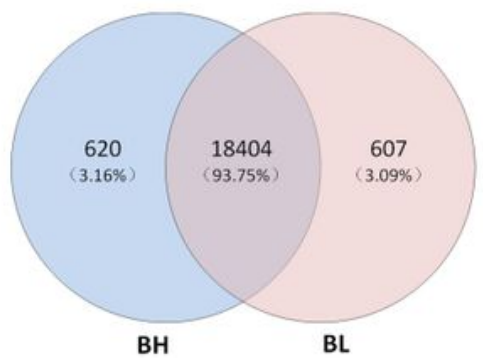

F

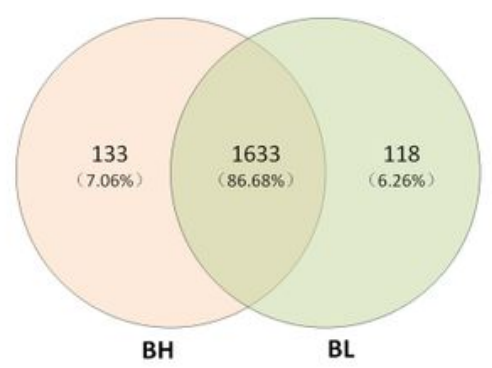

D

G
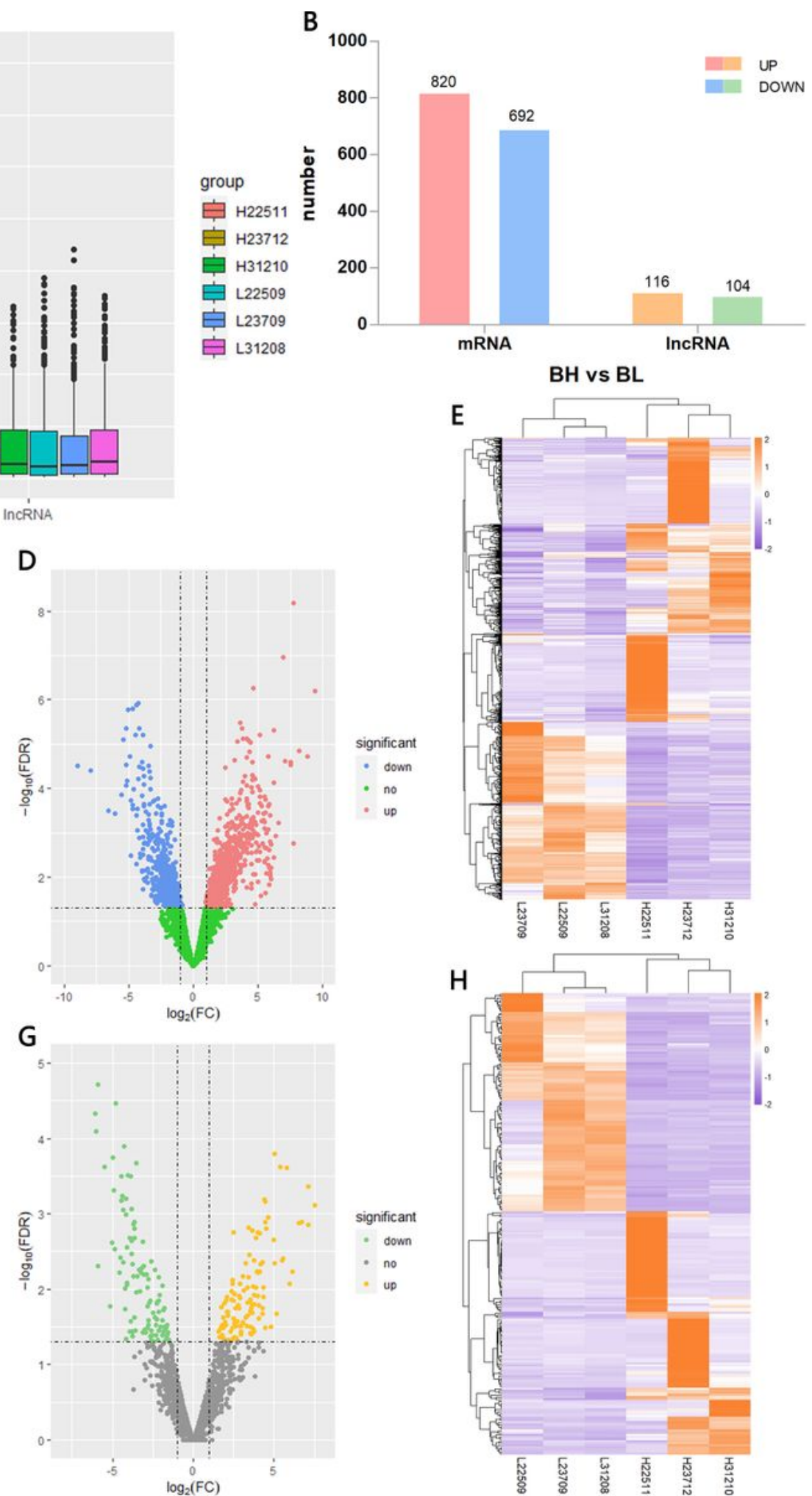
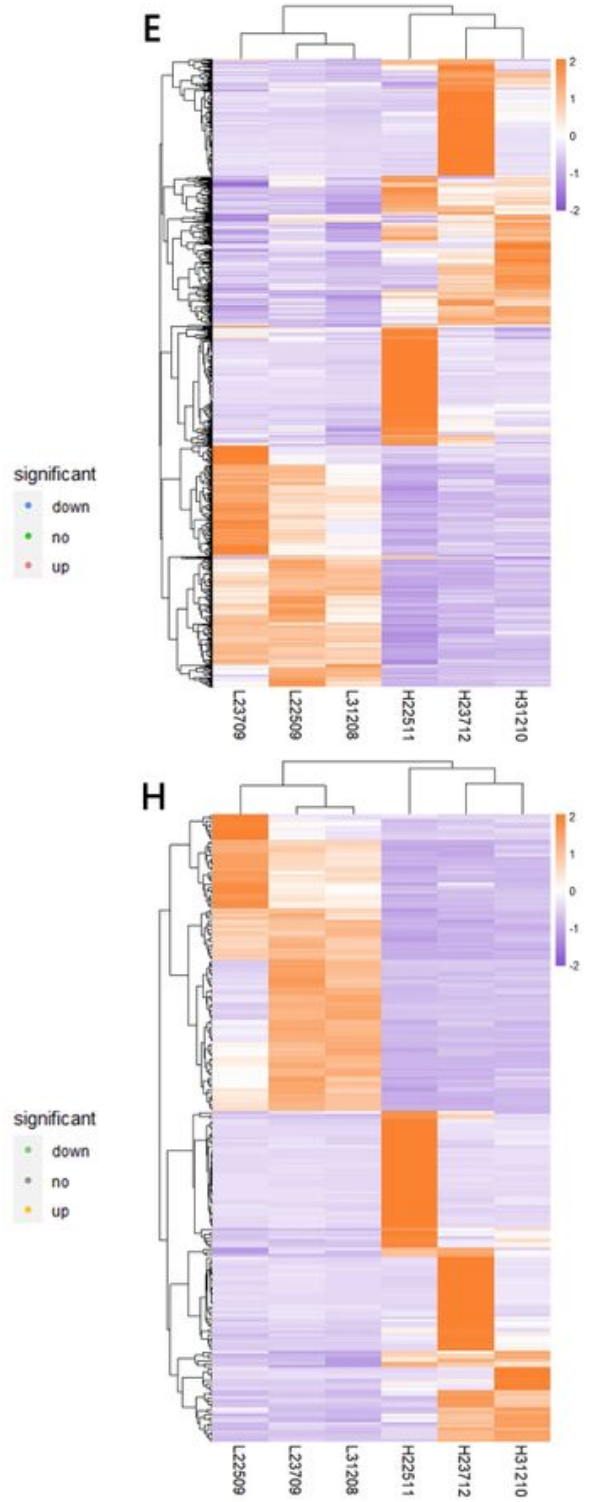

Figure 3

Figure 3 
A
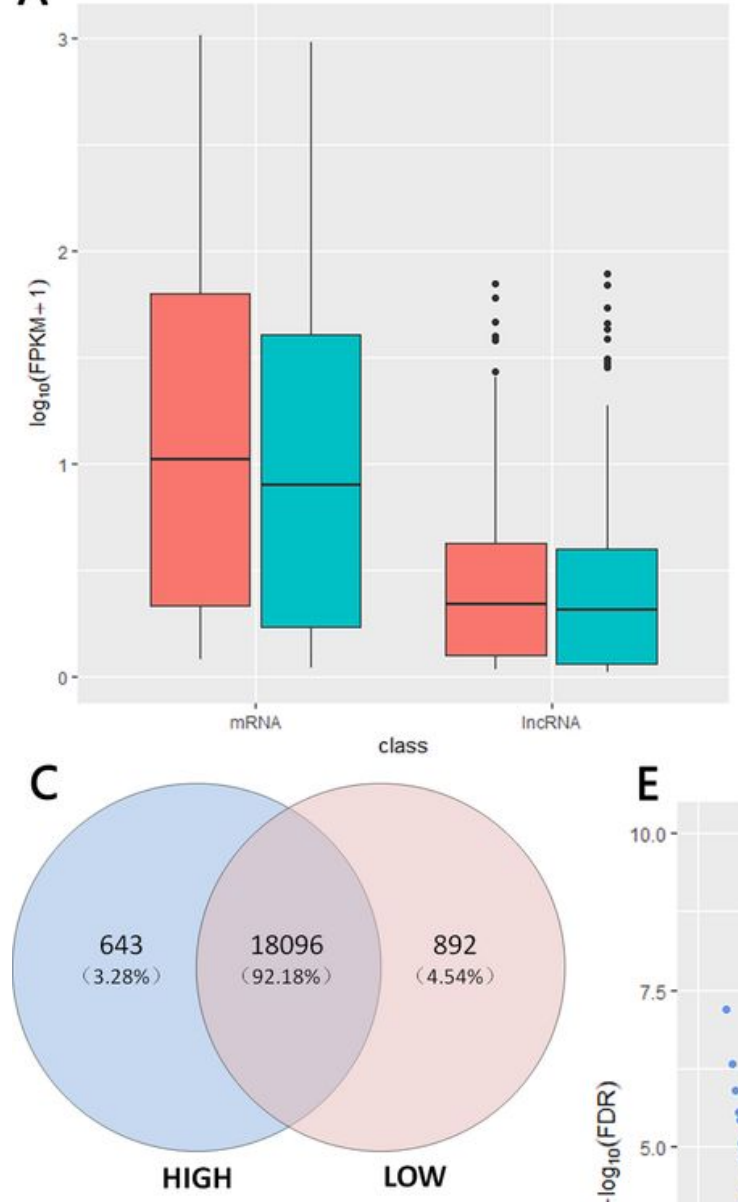

D
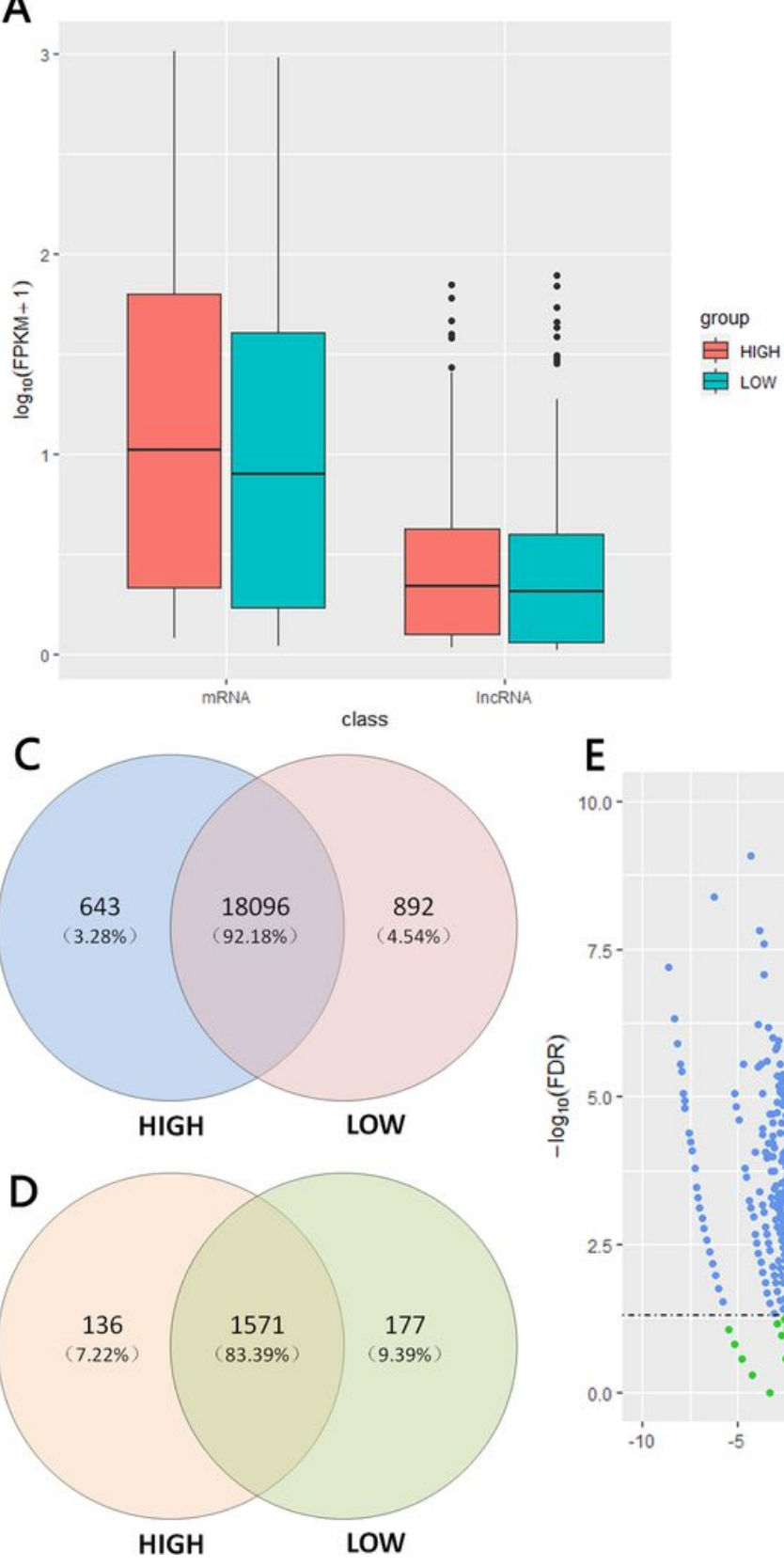

B

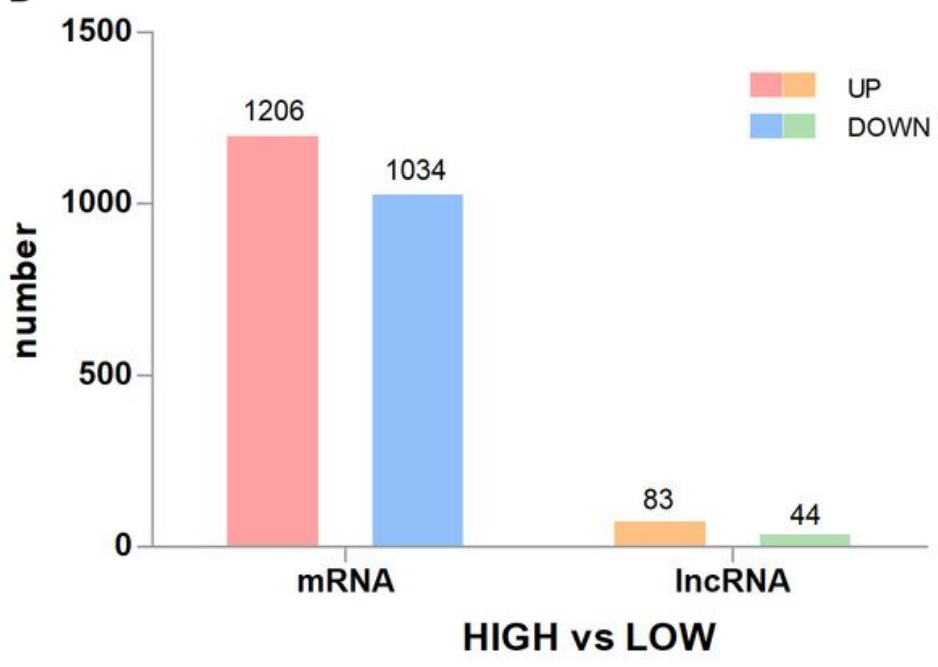

HIGH vs LOW

F

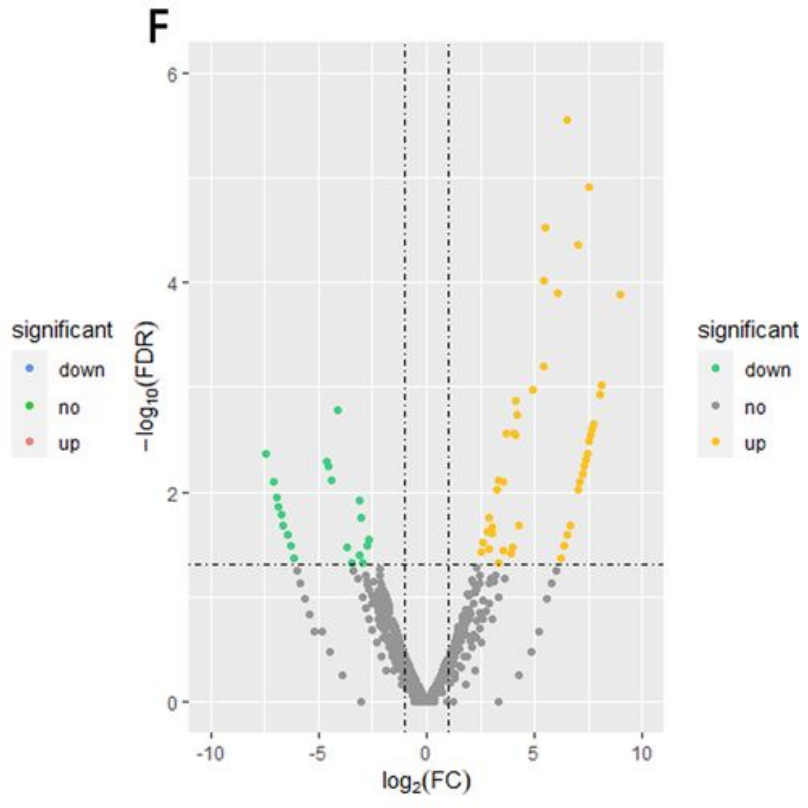

Figure 4

Figure 4 
A

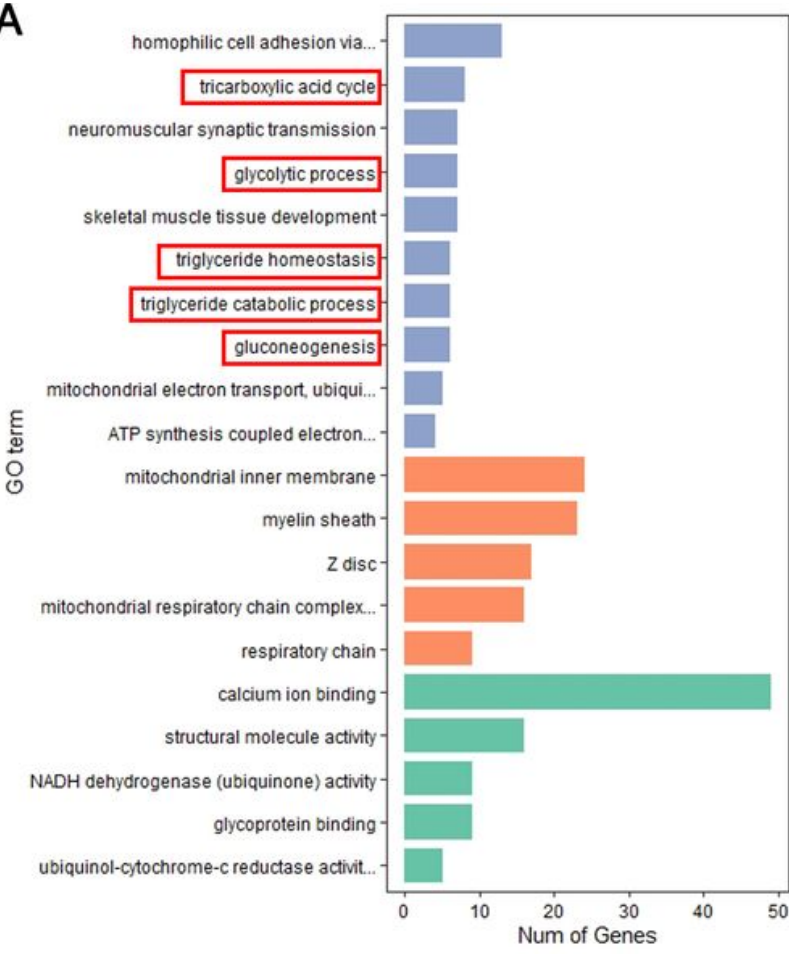

C

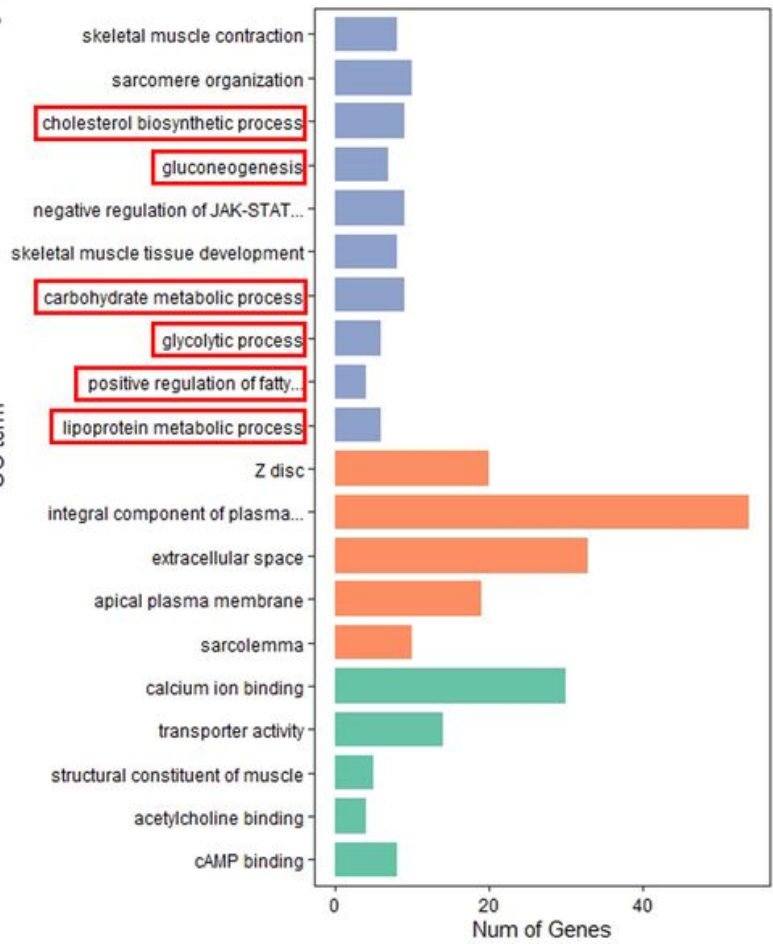

GO_Function

biological_process

cellular_component

molecular_function

GO_Function

biological_process

cellular_component

molecular_function
B

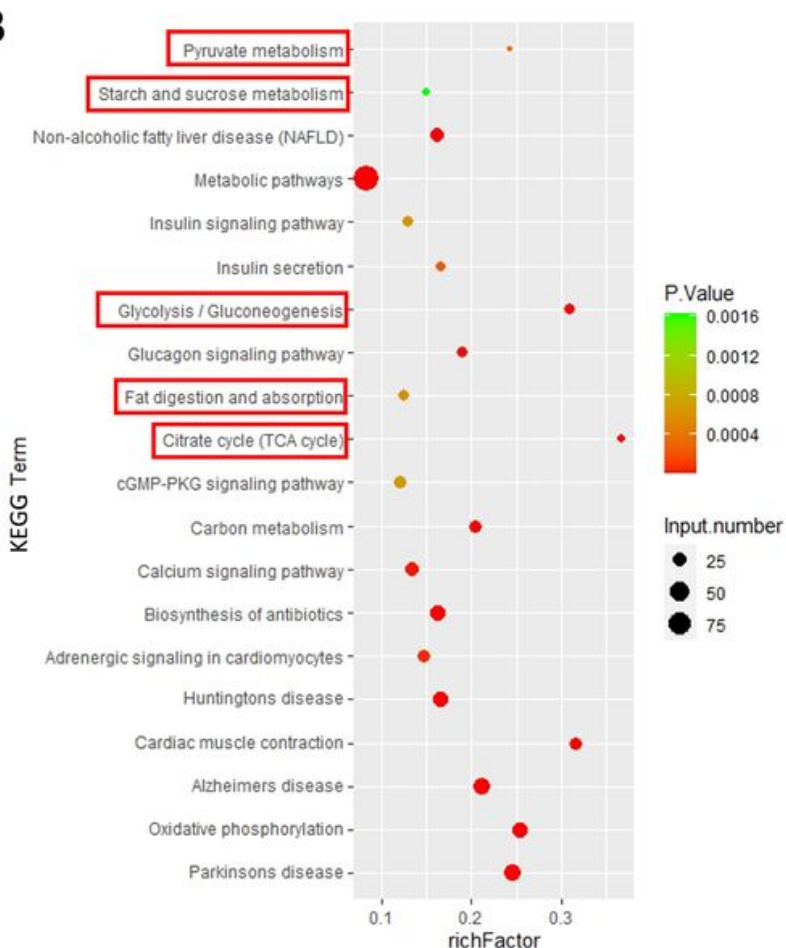

D

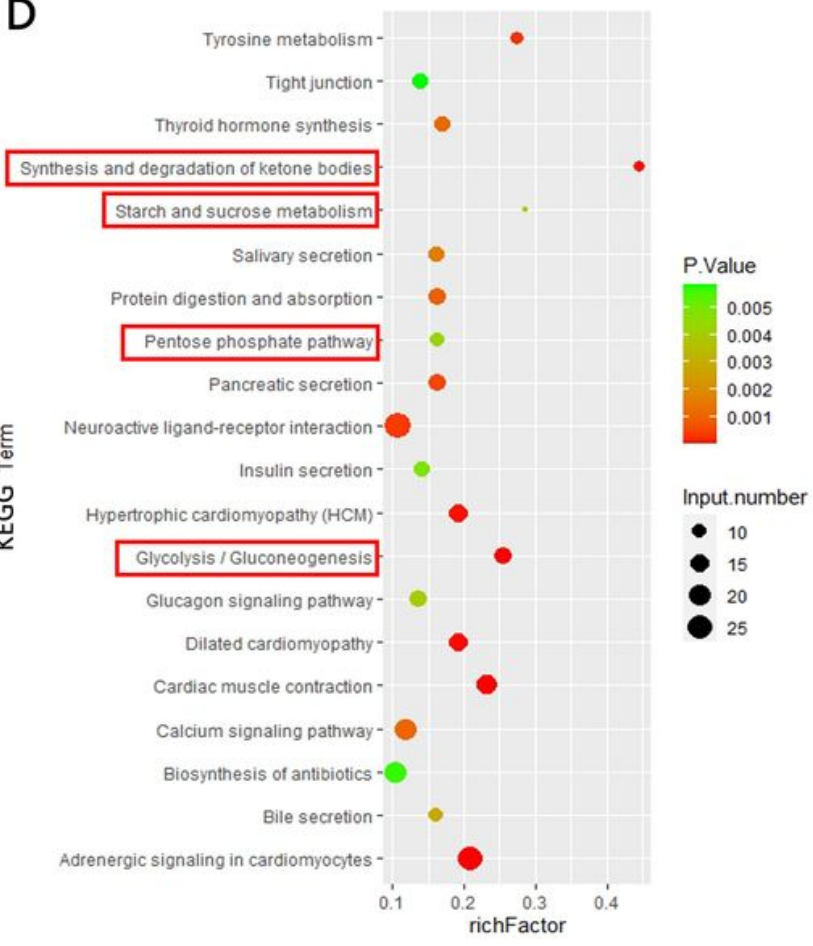

\section{Figure 5}

\section{Figure 5}




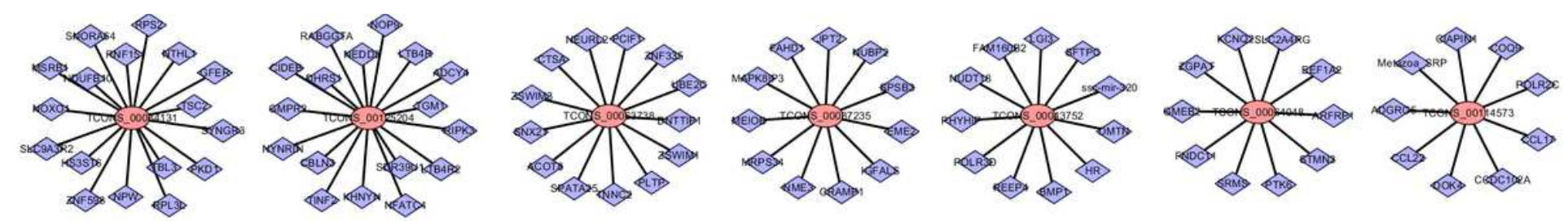

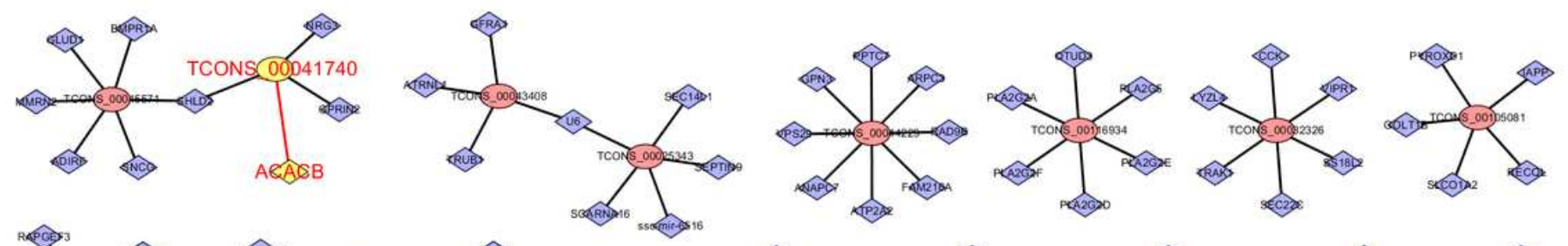

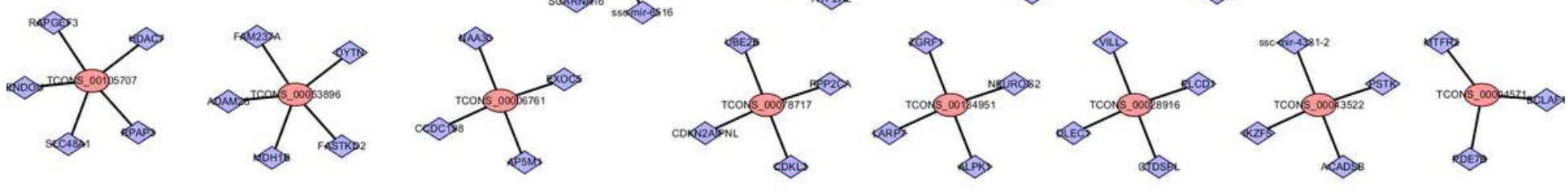

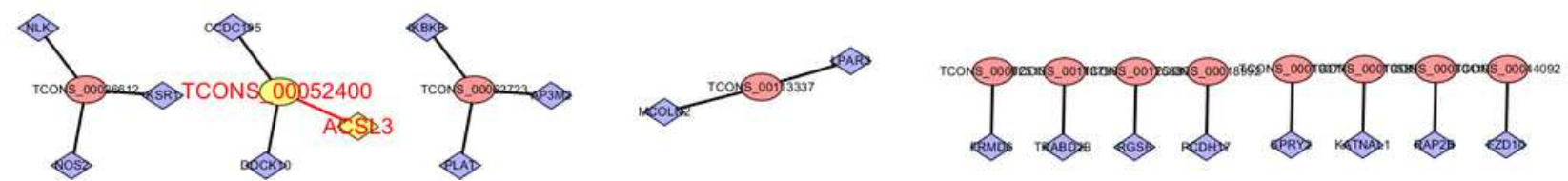

Figure 6

Figure 6 


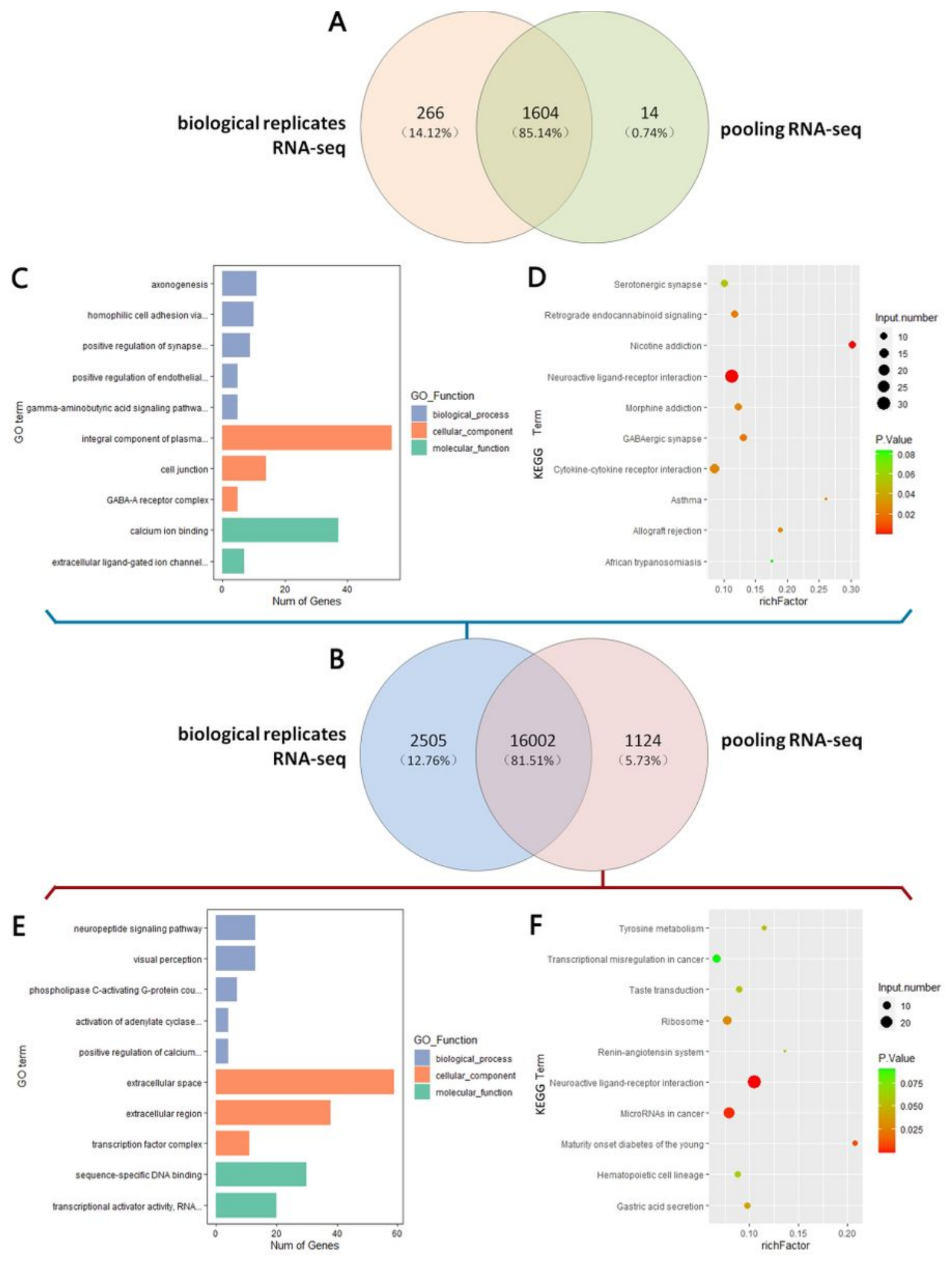

Figure 7

Figure 7

\section{Supplementary Files}

This is a list of supplementary files associated with this preprint. Click to download. 
- AdditionalFile3.xIsx

- AdditionalFile2.xIsx

- AdditionalFile1.pdf 